\title{
SIMULATION OF GUIDED WAVES IN CYLINDERS SUBJECT TO ARBITRARY BOUNDARY CONDITIONS USING THE SCALED BOUNDARY FINITE ELEMENT METHOD
}

\author{
Dominik Itner ${ }^{1}$, Hauke Gravenkamp ${ }^{1}$, Dmitrij Dreiling ${ }^{2}$, Nadine Feldmann ${ }^{2}$ AND Bernd \\ Henning $^{2}$ \\ ${ }^{1}$ Department of Civil Engineering, University of Duisburg-Essen \\ 45141 Essen, Germany \\ \{dominik.itner|hauke.gravenkamp\}@uni-due.de \\ ${ }^{2}$ Measurement Engineering Group, Paderborn University \\ 33098 Paderborn, Germany \\ \{dreiling|feldmann|henning\}@emt.uni-paderborn.de
}

Key words: ultrasound, guided waves, wave propagation, hierarchical shape functions, scaled boundary finite element method

\begin{abstract}
The scaled boundary finite element method (SBFEM) excels as a tool for numerical analysis at particular problem setups where the analytical solution in the scaling direction can be exploited to improve computational efficiency by reducing the number of required degrees of freedom (DOF). This is especially the case for simulating axisymmetric waveguides in the high-frequency range, allowing a significant decrease of computational costs (both memory and CPU time). Then, only the radial direction in a cylindrical coordinate system is discretized and the axial direction is solved analytically. A full threedimensional formulation is possible via the Fourier transform to include asymmetries. This contribution presents such an axisymmetric formulation, which is extended to allow the definition of circumferential as well as arbitrarily shaped dynamic boundary conditions (BCs). Furthermore, the required number of DOF depends on the frequency content. Hierarchical shape functions allow to dynamically adapt the DOF, further increasing efficiency. It will be shown that the results are in good agreement with standard finite element procedures, while greatly reducing computational time.
\end{abstract}

\section{INTRODUCTION}

Ultrasound simulations of soft materials such as polymers usually require a large number of DOF to properly capture the displacement solution when using standard FEM applications. This comes at the detriment of memory usage and computation times and is especially difficult to handle given long structures or unbounded domains. For such applications, the SBFEM has been proven a useful tool solving linear elastic problems in the frequency domain $[1,3]$.

When considering an axisymmetric domain along a cylindrical coordinate system, only the radial direction is discretized whereas an analytical solution is considered in the axial direction. Such a model can be extended in the SBFEM to include asymmetries in BCs allowing a full three-dimensional description of the problem by using concepts of the Fourier FEM. Then, the system is approximated w.r.t. the circum- 
ference by a truncated Fourier series and solved for each spatial frequency. Likewise, such a dynamic asymmetric BC must then be transformed, both in time and space. An arbitrarily shaped BC can vary with the radius and circumference and as such must be sampled at several distinct radii and transformed individually while still retaining computational efficiency.

In this paper, we briefly introduce the SBFEM for cylindric domains leading to the dynamic stiffness matrix. Subsequently, we present the preprocessing for applying an arbitrarily spatially varying BC; especially in the context of hierarchical shape functions. We verify the proposed approach by comparison with a finite element-model and demonstrate that a significant speed-up can be achieved for dynamic simulations.

\section{THE SBFEM FORMULATION}

A short summary of the cylindric SBFEM formulation is presented here. The displacement-strain relationship is decomposed into its constituent differentials and processed individually

$$
\epsilon=\mathbb{L} \mathbf{u}=\mathrm{b}_{1} \partial_{z} \mathbf{u}+\frac{1}{r} \mathbf{b}_{2} \partial_{\theta} \mathbf{u}+\mathrm{b}_{3} \partial_{r} \mathbf{u}+\frac{1}{r} \mathbf{b}_{4} \mathbf{u}=\mathbf{B}_{1} \partial_{z} \hat{\mathbf{u}}+\mathbf{B}_{2} \hat{\mathbf{u}}
$$

which leads to matrices $\mathbf{B}_{1}$ and $\mathbf{B}_{2}$ given polar number $m$ (circumferential frequency), additionally using a discretization with the matrix of shape functions $\mathbf{N}$ for the discrete displacement vector $\hat{\mathbf{u}}$ [2]:

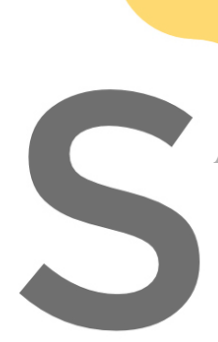

$$
\mathbf{B}_{1}=\mathbf{b}_{1} \mathbf{N} \quad \text { and } \quad \mathbf{B}_{2}=\frac{1}{r}\left(i m \mathbf{b}_{2}+\mathbf{b}_{4}\right) \mathbf{N}+\frac{2}{h} \mathbf{b}_{3} \partial_{\eta} \mathbf{N}, m \in \mathbb{N}_{0}
$$
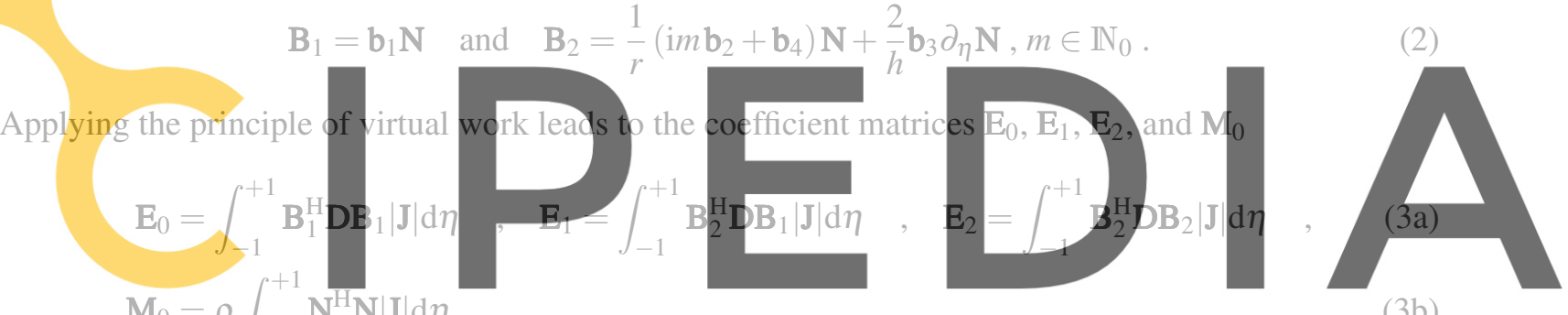

$$
\mathbf{M}_{0}=\rho \int_{-1}^{+1} \mathbf{N}^{\mathrm{H}} \mathbf{N}|\mathbf{J}| \mathrm{d} \eta
$$

(3b)

Register for free at https//www.scipedia.com to download the version without the watermark in the following partial differential equation:

$$
\mathbf{E}_{0} \partial_{z z} \hat{\mathbf{u}}+\left(\overline{\mathbf{E}}_{1}^{H}-\overline{\mathbf{E}}_{1}\right) \partial_{z} \hat{\mathbf{u}}-\overline{\mathbf{E}}_{2} \hat{\mathbf{u}}-\overline{\mathbf{M}}_{0} \partial_{t t} \hat{\mathbf{u}}=0 \quad \Rightarrow \quad\left[\lambda^{2} \overline{\mathbf{E}}_{0}+\lambda\left(\overline{\mathbf{E}}_{1}^{H}-\overline{\mathbf{E}}_{1}\right)-\overline{\mathbf{E}}_{2}-\omega^{2} \overline{\mathbf{M}}_{0}\right] \hat{\mathbf{u}}=0,
$$

which can be solved in the frequency domain with angular frequencies $\omega$ for eigenvalues $\lambda$. Likewise, the coefficient matrix $\mathbf{Z}$ with

$$
\mathbf{Z}(m, \omega)=\left[\begin{array}{cc}
\mathbf{E}_{0}^{-1} \mathbf{E}_{1}^{\mathrm{H}} & -\mathbf{E}_{0}^{-1} \\
\omega^{2} \mathbf{M}_{0}+\mathbf{E}_{1} \mathbf{E}_{0}^{-1} \mathbf{E}_{1}^{\mathrm{H}}-\mathbf{E}_{2} & -\mathbf{E}_{1} \mathbf{E}_{0}^{-1}
\end{array}\right]
$$

can be derived, leading to the ordinary matrix differential equation and its general solution [2]

$$
\partial_{z} \boldsymbol{\phi}(z)=-\mathbf{Z} \boldsymbol{\phi}(z) \Rightarrow \boldsymbol{\phi}(z)=\Psi \mathrm{e}^{\Lambda z} \mathbf{c} \quad \text { with } \quad \boldsymbol{\phi}(z)=\left[\begin{array}{l}
\hat{\mathbf{u}}(z) \\
\hat{\mathbf{q}}(z)
\end{array}\right]
$$

to the eigenvalue problem $-\mathbf{Z} \Psi=\boldsymbol{\Psi} \Lambda$. Here, $\Psi$ is the eigenvector matrix associated with the diagonal eigenvalue matrix $\Lambda$, and $\mathrm{c}$ is the vector of integration constants. Partitioning the solution in Eq. (6) 
leads to the dynamic stiffness matrix of a bounded domain of arbitrary length $L$ [3] of a solid or hollow cylinder

$$
\mathbf{S}_{L}(m, \omega)=\left[\begin{array}{cc}
-\Psi_{\mathrm{qp}} & -\boldsymbol{\Psi}_{\mathrm{qn}} \mathrm{e}^{-\Lambda_{\mathrm{n}} L} \\
\boldsymbol{\Psi}_{\mathrm{qp}} \mathrm{e}^{\boldsymbol{\Lambda}_{\mathrm{p}} L} & \boldsymbol{\Psi}_{\mathrm{qn}}
\end{array}\right]\left[\begin{array}{ll}
\boldsymbol{\Psi}_{\mathrm{up}} & \boldsymbol{\Psi}_{\mathrm{un}} \mathrm{e}^{-\Lambda_{\mathrm{n}} L} \\
\boldsymbol{\Psi}_{\mathrm{up}} \mathrm{e}^{\Lambda_{\mathrm{p}} L} & \boldsymbol{\Psi}_{\text {un }}
\end{array}\right]^{-1},
$$

which is given for each combination of $\omega$ and $m$ and defines the relationship between displacements and external forces at the opposing cylinder faces:

$$
\left[\begin{array}{l}
\left.\hat{\mathbf{p}}(m, \omega)\right|_{z=0} \\
\left.\hat{\mathbf{p}}(m, \omega)\right|_{z=L}
\end{array}\right]=\mathbf{S}_{L}(m, \omega)\left[\begin{array}{l}
\left.\hat{\mathbf{u}}(m, \omega)\right|_{z=0} \\
\left.\hat{\mathbf{u}}(m, \omega)\right|_{z=L}
\end{array}\right] .
$$

Boundary conditions may then be prescribed along the discretized flat surfaces either at $z=0$ or $z=L$ and the linear equation system in Eq. (8) solved as per usual.

\section{ARBITRARILY SHAPED BOUNDARY CONDITION}

Given that the stiffness matrix and consequently the system of linear equations is solved in the frequency domain, any dynamic BC with an asymmetric circumferential distribution must be similarly Fourier transformed $F(m, \omega)=\mathscr{F}(f(\theta, t))$. In the trivial case, the distribution of a BC may be assumed to have following property:

$$
f(r, \theta, t)=f(r) \cdot f(\theta) \cdot f(t),
$$

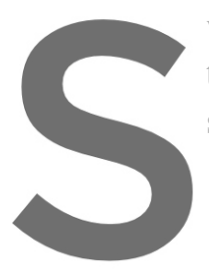

which allows to indivicur

tively, ${ }^{1}$ as well as integ shaped BCs lose the ind
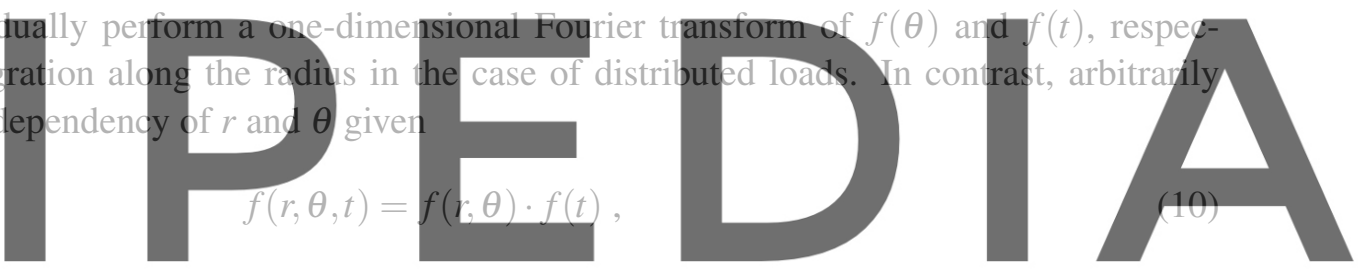

introducing a relationship between the radial and circumferential distribution, i.e. an arbitrarily shaped

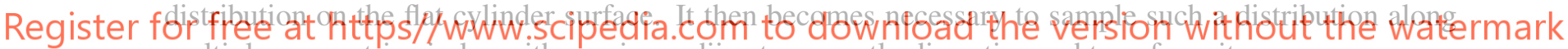
multiple concentric circles with varying radii $r_{j}$ to correctly discretize and transform it:

$$
F\left(r_{j}, m\right)=\frac{1}{2 \pi} \int_{0}^{2 \pi} f\left(r_{j}, \theta\right) \cdot \mathrm{e}^{-\mathrm{i} m \theta} \mathrm{d} \theta, m \in \mathbb{N}_{0} .
$$

Each concentric distribution is independently Fourier-transformed to finally assemble the vector of discrete values for a particular polar number $m$ (and angular frequency $\omega$ ):

$$
\hat{\mathbf{f}}=\left[\begin{array}{lllll}
\hat{f}_{1} & \ldots & \hat{f}_{j} & \ldots & \hat{f}_{n}
\end{array}\right]^{\mathrm{T}} \quad \text { with } \quad \hat{f}_{j}=F\left(r_{j}, m\right) \cdot F(\omega) .
$$

The radii $r_{j}$ are chosen to coincide with the position of nodes if the discretization is based on shape functions that possess the Kronecker delta property. It may also be necessary to select the integration points, instead, depending on the integration scheme for loads. However, using hierarchical shape functions, a more involved process is required, as the discrete values are not related to physical positions but rather represent the amplitude spectrum of the polynomial basis functions and hence require conversion. Then,

\footnotetext{
${ }^{1}$ Due to symmetry reasons, the number of coefficients for which the system must be solved is halved, not quartered.
} 

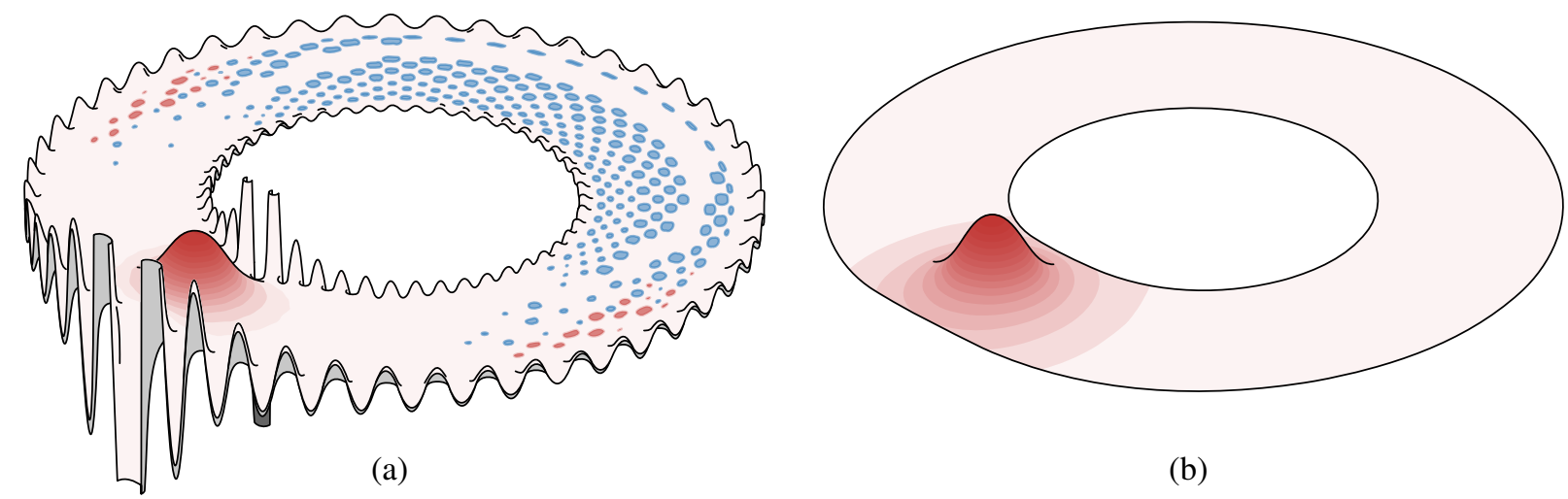

(b)

Figure 1: The displacement solution to the static case for a Neumann boundary condition demonstrates the error comparing an insufficient equidistant sampling (a) and "well-behaved" sampling using GaussLobatto-Legendre points (b).

the choice of $r_{j}$ is not trivial and can introduce errors in the approximation and therefore the solution. These errors are expressed as edge effects which originate in Runge's phenomenon (as circumvented by spectral elements) and especially occur using equidistant positions [2, 4]; additionally, these amplifications of interpolated values are smeared along the circumference due to the Fourier transform, cf. Fig. 1a and Fig. 1b. As such, an adequate choice of radii $r_{j}$ is required.

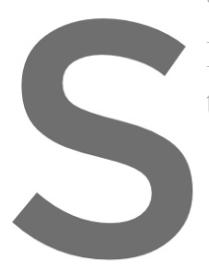

It was found that when tions, with

Register for free at https//www.scipedia. $\frac{2 b m^{1} t}{2} \phi_{-1}^{\eta} d o w n k l g a d$ the kersion without the watermark

(here, $L_{k-1}$ refers to the Legendre polynomials), then likewise Gauss-Lobatto-Legendre points provide a good choice to circumvent Runge's phenomenon and minimize the approximation error. A mapping between the physical domain and the polynomial coefficient space can be constructed by evaluating the Lobatto polynomials at local coordinates $\eta_{j}$ that correspond to $r_{j}$ with $n=p+1$

$$
\hat{\mathbf{N}}=\left[\begin{array}{ccccc}
N_{1}\left(\eta_{1}\right) & \ldots & N_{k}\left(\eta_{1}\right) & \ldots & N_{n}\left(\eta_{1}\right) \\
\vdots & & \vdots & & \vdots \\
N_{1}\left(\eta_{j}\right) & \ldots & N_{k}\left(\eta_{j}\right) & \ldots & N_{n}\left(\eta_{j}\right) \\
\vdots & & \vdots & & \vdots \\
N_{1}\left(\eta_{n}\right) & \ldots & N_{k}\left(\eta_{n}\right) & \ldots & N_{n}\left(\eta_{n}\right)
\end{array}\right]
$$

and subsequently inverting the matrix $\hat{\mathbf{N}}$ :

$$
\hat{\mathbf{f}}(m)=\hat{\mathbf{N}}^{-1} F(\mathbf{r}, m) \quad, \quad \mathbf{r}^{\mathrm{T}}=\left[\begin{array}{lllll}
r_{1} & \ldots & r_{j} & \ldots & r_{n}
\end{array}\right] .
$$




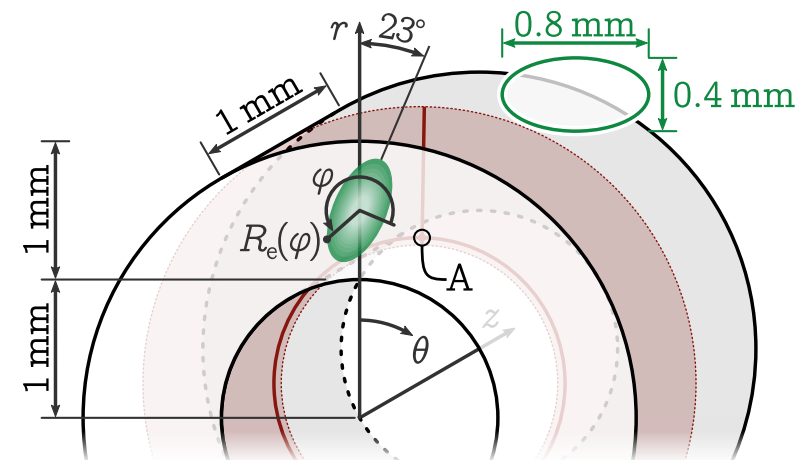

Figure 2: Geometry of hollow cylinder. Load is applied on ellipse area. Displacements $u_{z}$ are fixed at the base. Sample point of interest is indicated in point A with $r=1.05 \mathrm{~mm}$ and $\theta=1^{\circ}$

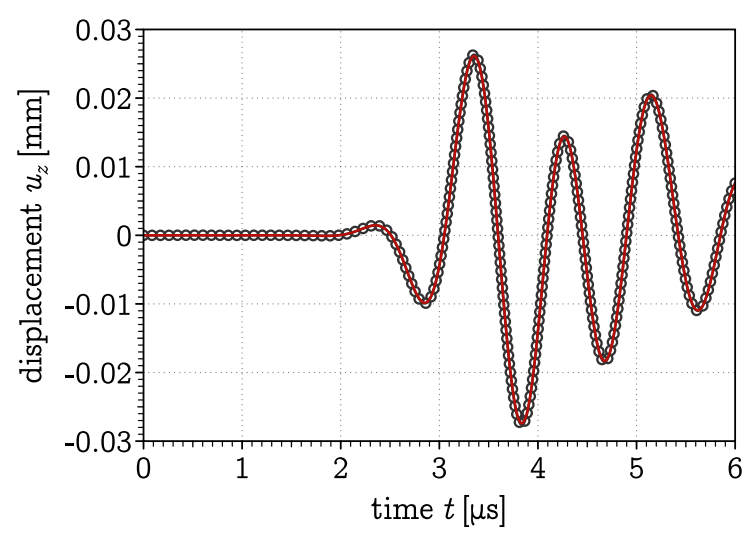

Figure 3: Dynamic response $u_{z}$ of FEM (-) and SBFEM $(O)$ in point A shown in Fig. 2.

The matrix $\hat{\mathbf{N}}$ is not strictly required to be a square matrix, however, undersampling is not recommended. In case of oversampling, the pseudoinverse must necessarily be applied. When external forces are prescribed, additional pre-integration of shape functions is necessary to assemble a Neumann BC:
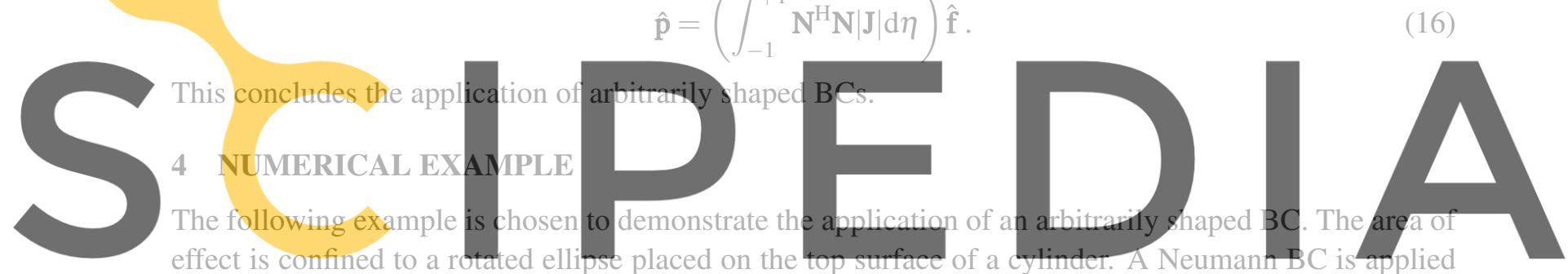

given a parabolic distribution around the center of the ellipse in the local coordinate system $(R, \varphi)$, see

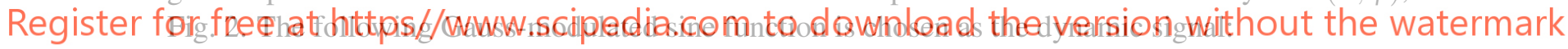

$$
f(t)=\sin (2 \pi \cdot 1 \mathrm{MHz} \cdot t) \cdot \exp \left(-\frac{1}{2}\left[\frac{t-3 \mu \mathrm{s}}{0.5 \mu \mathrm{s}}\right]^{2}\right) \cdot(1 \mathrm{GPa}) .
$$

Additionally, displacements in $z$ are fixed at the bottom. The displacements are evaluated at $z=0.5 \mathrm{~mm}$ and the $\mathrm{L}^{2}$-norm is applied to compute the overall deviation averaged in space and time. As reference the solution aquired by Ansys 2019 R 2 is chosen using implicit time integration. There, the mesh is defined by an average element size of $0.1 \mathrm{~mm}$ (roughly 10 elements in $r, z$ and 128 elements in $\theta$ ), and a time step of $0.04 \mu \mathrm{s}$ with a time window of interest of $6 \mu \mathrm{s}$. For the convergence study, each value is halved for a total of four simulations. The SBFEM model, on the other hand, requires only a minimum and maximum number of 15 and 17 DOF per discretized edge, respectively, to reach a precision comparable to the reference. Here, we apply an adaptive algorithm to automatically pick the number of DOF depending on the current frequencies $\omega$ and $m$, maintaining a predefined error. However, the minimum number is increased to properly capture the BC.

Selecting 5289 unique Fourier coefficients to compute the solution, a computing time of $89.4 \mathrm{~s}$ is required and yields a speedup of over 2000 compared to the third Ansys solution (mesh size: $0.025 \mathrm{~mm}$, 


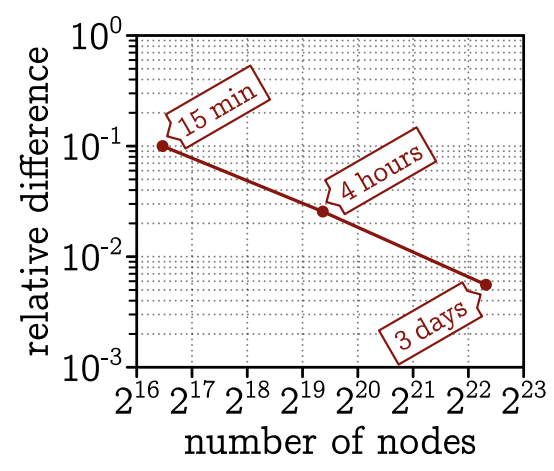

(a)

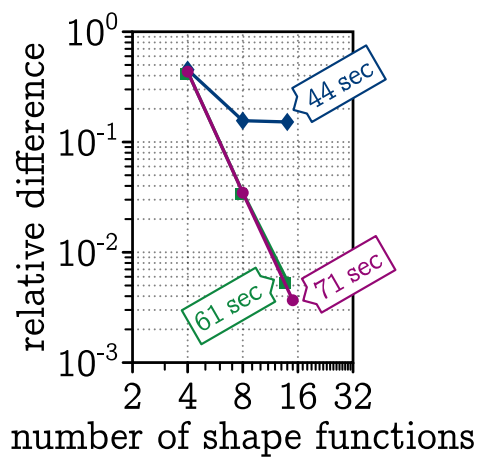

(b)

Figure 4: Convergence behavior of FEM (a) using $h$-refinement and SBFEM (b) using $p$-refinement for $2952(\$), 4428(-)$, and 5289 ( $)$ Fourier coefficients. Computation times are indicated. Both solutions use a fourth FEM simulation as reference (with roughly seven weeks computation time).

time step: $0.01 \mu \mathrm{s}$ ). Relative differences of $0.8684 \%, 0.9107 \%$, and $0.8369 \%$ in $r, \theta$, and $z$, respectively, can be found between the methods. Figure 3 shows the dynamic response in $u_{z}$ at the single point indicated in Fig. 2, which possesses the largest relative deviation of all sample points with $0.8090 \%$ for the simulated time frame. As can be seen, the solutions are in very good agreement.

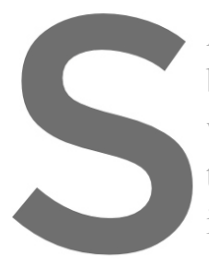

A more thorough compa

but also due to truncation

when the convergence for

the previously mentioned

is allowed. (This explains
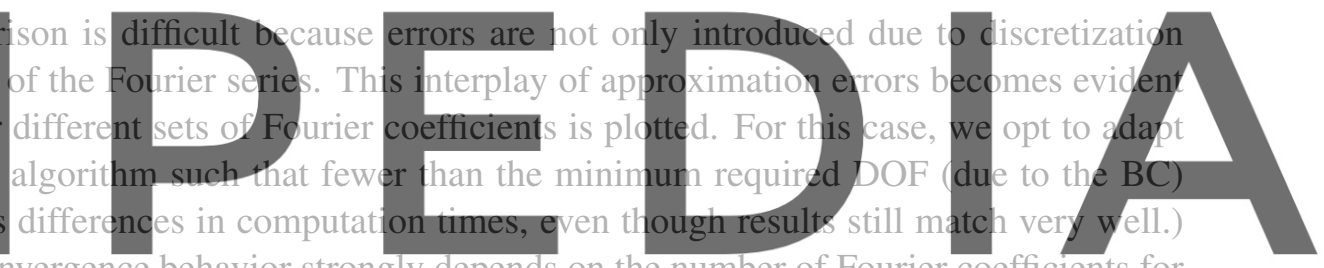

As can be seen, proper convergence behavior strongly depends on the number of Fourier coefficients for

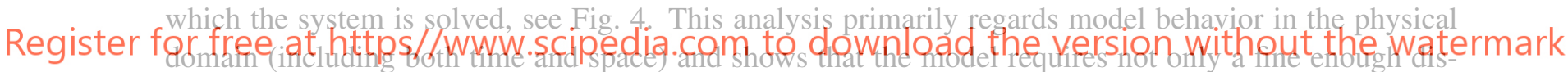
cretization but also enough information in the frequency domain to capture the solution well enough in the physical domain.

\section{CONCLUSION}

In this contribution, the cylindric SBFEM was presented and arbitrarily shaped BC were incorporated. It was demonstrated that the formulation gives good results while requiring only a fraction of the computing time of conventional FEM applications.

Acknowledgement Funded by the Deutsche Forschungsgemeinschaft (DFG, German Research Foundation) 409779252. 


\section{REFERENCES}

[1] Song, C. and Wolf, J. P. The scaled boundary finite-element method—alias consistent infinitesimal finite-element cell method-for elastodynamics. Computer Methods in Applied Mechanics and Engineering (1997) 147(3):329-355.

[2] Gravenkamp, H. and Birk, C. and Song, C. The computation of dispersion relations for axisymmetric waveguides using the Scaled Boundary Finite Element Method. Ultrasonics (2014) 54(5):13731385 .

[3] Gravenkamp, H. and Birk, C. and Song, C. Simulation of elastic guided waves interacting with defects in arbitrarily long structures using the Scaled Boundary Finite Element Method. Journal of Computational Physics (2015) 295:438-455.

[4] Vu, T.H. and Deeks, A.J. Use of higher-order shape functions in the scaled boundary finite element method. Int. J. Numer. Methods Eng. (2006) 65(10):1714-1733.
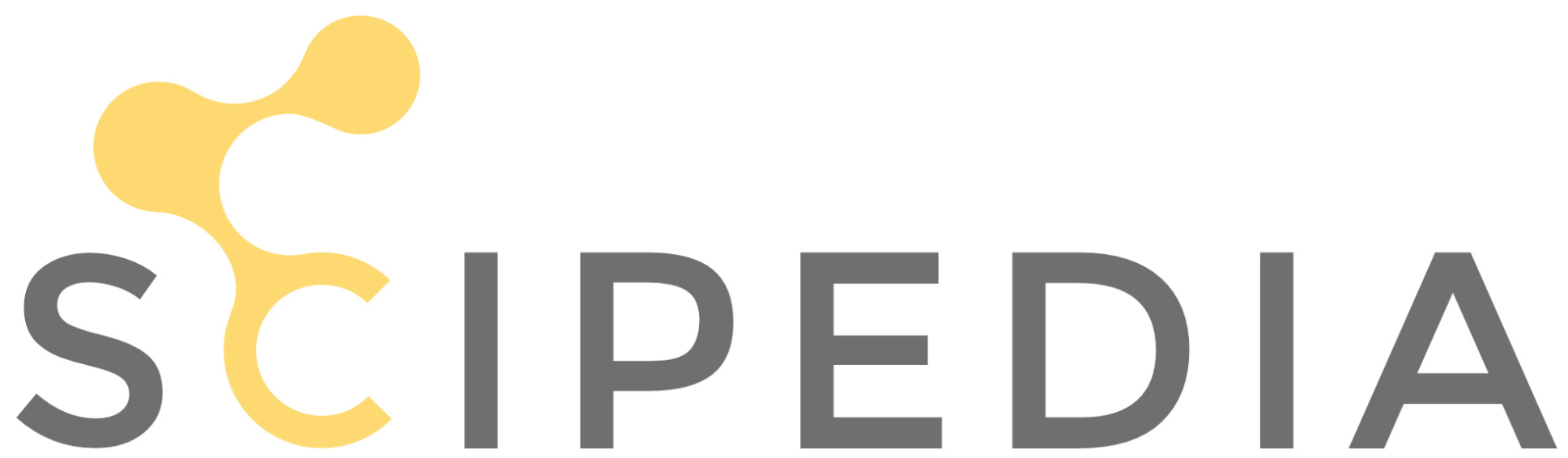

Register for free at https//www.scipedia.com to download the version without the watermark 